This is a postprint version of the following published document:

Valencia, J., Baselga, J. \& Piérola, I. F. (2009).

Compression elastic modulus of neutral, ionic, and amphoteric hydrogels based on N-vinylimidazole.

Journal of Polymer Science Part B: Polymer Physics, 47

(11), pp. 1078-1087.

DOI: $10.1002 /$ polb.21712

(C) Wiley, 2009 


\title{
Compression Elastic Modulus of Neutral, Ionic, and Amphoteric Hydrogels Based on N-Vinylimidazole
}

\author{
JOAQUIN VALENCIA, ${ }^{1}$ JUAN BASELGA, ${ }^{2}$ INES F. PIEROLA ${ }^{1}$ \\ ${ }^{1}$ Departamento de Ciencias y Técnicas Fisicoquímicas, Facultad de Ciencias, Universidad a Distancia (UNED), c/ Senda \\ del Rey 9, 28040 Madrid, Spain \\ ${ }^{2}$ Departamento Ciencia e Ingeniería de Materiales e Ingeniería Química, Universidad Carlos III, 28911 Leganés, Spain
}

\begin{abstract}
Several hydrogels of $\mathrm{N}$-vinylimidazole and sodium styrenesulfonate have been prepared by radical cross-linking copolymerization in aqueous solution, using $\mathrm{N}, \mathrm{N}^{0}$-methylene-bisacrylamide as crosslinker. Depending on composition, these hydrogels were neutral, amphoteric, cationic or anionic. Compression-strain measure-ments were performed on samples as-synthesized and swollen in deionized water or in acid aqueous solutions, with and without salt. It was thus found that the cross-linking densities determined by compression measurements on assynthesized sam-ples are in good accordance with those calculated by means of the model of polymer networks with pendant vinyl groups. A non-Gaussian parameter (b) was introduced to explain that the elastic moduli (G) of samples swollen at equilibrium are larger than predicted by the Gaussian model. The $b$ values of the neutral or ionized systems increase with swelling and fall into a single curve, which denotes a common behav-ior. Swelling has two opposite effects on $G$; on the one hand $G$ decreases because the polymer volume fraction diminish and the system shifts from the affine limit to the phantom one; on the other, $b$ increases and contributes to increasing $G$. The balance of those two opposite effects determines the variation of $\mathrm{G}$ with swelling. The possi-ble contribution of ionic crosslinks to $\mathrm{me}_{\mathrm{e}}$ for the polyampholyte and for the polycation wearing divalent counteranions was discussed. A peculiar system is poly(sodium styrenesulfonate), whose cross-linking density is much lower than expected.
\end{abstract}

\section{KEYWORDS}

compression; crosslinking; degree of crosslinking; elastic modulus; hydrogels; ionic gels; modulus; networks; polyelectrolytes

\section{INTRODUCTION}

Cross-linked polymers exhibit interesting proper-ties, many of which depend on the degree of cross-linking. The cross-linking density $\left(\mathrm{m}_{\mathrm{e}}\right)$ can be determined by means of different techniques such

as swelling measurements, ${ }^{1-5}$ stress-strain $^{6-8}$ or compression-strain measurements, ${ }^{9-16}$ dynamic mechanic analysis, ${ }^{17}$ calorimetric measurements of the glass transition temperature, ${ }^{5,18,19}$ and spectroscopic studies (Raman, NMR) ${ }^{20}$ or chemi-cal elemental analysis. ${ }^{21}$ The physical meaning of the $m_{e}$ values determined through each technique is different and therefore the results obtained are also slightly different. For example, the perma-nent cross-linking density determined by $\mathrm{Tg}$ measurements represents the covalent junctions 
between chains that contribute to immobilize chains or to diminish free volume. This includes the knots joined to elastically effective chains and those connected to network defects (dangling chains, intramolecular cycles). ${ }^{5,18}$ The same holds for $v_{\mathrm{e}}$ determined spectroscopically or chemically. On the contrary, the effective cross-linking density determined through swelling measurements reflects not only permanent junctions but also morphological features such as the inherent porosity. ${ }^{5}$ Finally, the $v_{\mathrm{e}}$ determined through mechanical properties represents the density of knots joined only to elastically effective chains and therefore contributing to the elastic component of the osmotic swelling pressure. The crosslinking density of hydrogels is difficult to be measured through mechanical measurements because xerogels usually are extremely rigid and swollen hydrogels very often lack of the necessary mechanical integrity. ${ }^{6,9,22-24}$ Even though, whenever possible, compression or stress-strain measurements on swollen samples are preferred to determine $v_{\mathrm{e}}$ of polymer networks.

The correct determination of $v_{\mathrm{e}}$ is decisive to understand the role of the elastic component of the osmotic swelling pressure in the behavior of cross-linked polymers. ${ }^{3,4}$ Hydrogels based on $N$ vinylimidazole (VI) may be particularly useful in that respect because they have been broadly studied and the parameters necessary to perform quantitative analysis are likely known..$^{3-5,18,25-34}$ Besides, they show many different effects with different comonomers. For instance, swelling measurements on cross-linked copolymers with sodium styrenesulfonate (SS) and VI (PVI-SS) show a pronounced overshoot, namely, the swelling degree of samples with small SS content goes from zero to a maximum value (which can be very large) and again to almost zero, without external stimulus. ${ }^{30}$ Cross-linked copolymers of VI and several acrylamides or $N$-vinylcaprolactam are both $\mathrm{pH}$ and temperature responsive. ${ }^{31-34}$ Their phase transition temperatures increase with the degree of ionization of VI, which in turn depends on the $\mathrm{pH}$ of the medium.

Basic imidazole rings become protonated in acidic media, ${ }^{4,26-28}$ and as a consequence, neutral or cationic hydrogels can be obtained by swelling the same sample in neutral or acidic solutions. Besides, PVI-SS gels are anionic or amphoteric systems (depending on the SS content) because of VI protonation. ${ }^{3,29,30}$ Other amphoteric gels were obtained by cross-linking copolymerization of VI and acrylic acid. ${ }^{31}$ The degree of swelling of PVI-
SS shows minimum values for low SS contents and such minimum values increase with ionic strength. ${ }^{29}$ By contrast, PVI shows maximum swelling values for intermediate degrees of ionization. The $\mathrm{pH}$ of maximum swelling of PVI depends on the gel effective concentration, on the nature of the protonating acid and on the ionic strength. ${ }^{4,26}$ Divalent anions give place to lower swelling of PVI, while divalent cations do not influence swelling more than univalent cations in solutions with the same ionic strength. ${ }^{26}$

It was also found that $v_{\mathrm{e}}$ of chemically crosslinked poly( $N$-vinylimidazole) (PVI) depends on the comonomer concentrations in the feed and the conversion attained in the polymerization. ${ }^{18,25}$ For total conversion samples, $v_{\mathrm{e}}$ is proportional to the product of the total comonomer concentration and the cross-linker concentration. ${ }^{5,18,25}$ With increasing conversion, the permanent cross-linking density decreases ${ }^{18}$ while the effective crosslinking density (determined through swelling measurements) increases and changes inversely with inherent porosity. ${ }^{5}$

The aim of this work is to compare the elastic response of neutral, partially protonated PVI and PVI-SS gels. Cross-linked poly(sodium styrenesulfonate) (PSS) is also studied as a term for comparison. The contributions to the elastic modulus coming from ionic crosslinks and non-Gaussian effects are analyzed.

\section{EXPERIMENTAL}

\section{Synthesis of the Hydrogels}

Chemically cross-linked poly( $N$-vinylimidazole $)$ (PVI), poly(sodium styrenesulfonate) (PSS) and poly $(N$-vinylimidazole-co-sodium styrenesulfonate) (PVI-SS) hydrogels were synthesized by radical polymerization in aqueous solution with $N, N^{\prime}$-methylene-bisacrylamide (BA) as crosslinker. The initiator employed was potassium persulfate $(0.007 M)$ or benzoyl-peroxide $(0.035 M)$ for two different specimens of PSS and 2,2'-azobis(isobutyronitrile) (AIBN) for any other sample $\left(6 \times 10^{-3}-11 \times 10^{-3}\right.$ M). $N$-vinylimidazole (Aldrich) was distilled under reduced pressure at $55{ }^{\circ} \mathrm{C}$ just prior use. Water was distilled and deionized by a Milli-Q system from Millipore. Sodium styrenesulfonate (Aldrich), BA (Aldrich), benzoyl-peroxide (Merck), potassium persulfate (Probus) and AIBN (Fluka) were used as received.

An example of protocol employed in synthesis is the following: the aqueous solution of 
Table 1. Feed Comonomer Concentrations Employed in the Synthesis of Several Specimens of Samples PVI25(2), PVI40(2), PSS40(2), and PVI-SS40(2)4, Polymer Volume Fraction of the As-Synthesized Specimens $\left(v_{2 \mathrm{r}}\right)$ and Conversion of the Reaction of Polymerization $(\gamma)$

\begin{tabular}{ccccccc}
\hline Sample & Specimen & $\begin{array}{c}{[\mathrm{VI}]} \\
(\mathrm{mol} / \mathrm{L})\end{array}$ & $\begin{array}{c}{[\mathrm{SS}]} \\
(\mathrm{mol} / \mathrm{L})\end{array}$ & $\begin{array}{c}{[\mathrm{BA}]} \\
(\mathrm{mol} / \mathrm{L})\end{array}$ & $v_{2 \mathrm{r}}$ & $\gamma(\%)$ \\
\hline PVI25(2) & S9 & 2.6 & - & 0.03 & 0.128 & 62 \\
& S28 & 2.63 & & 0.034 & 0.183 & 82 \\
& S29 & 2.65 & & 0.033 & 0.188 & 86 \\
& S30 & 2.62 & & 0.035 & 0.177 & 81 \\
PVI40(2) & S31 & 2.64 & & 0.032 & 0.169 & 77 \\
& S3 & 4.1 & - & 0.05 & 0.283 & 85 \\
& S25 & 4.16 & & 0.052 & 0.273 & 81 \\
PSS40(2) & S26 & 4.22 & & 0.054 & 0.305 & 88 \\
& S27 & 4.21 & & 0.052 & 0.281 & 90 \\
PVI-SS40(2)4 & S8 & - & 1.75 & 0.062 & 0.250 & 96 \\
& S13 & & 1.71 & 0.058 & 0.246 & 92 \\
& S23 & 3.85 & 0.16 & 0.057 & 0.152 & 48
\end{tabular}

AIBN was employed as initiator for any sample except for PSS40(2) where benzoylperoxide (*specimen 8$)$ or potassium persulfate (**specimen 13$)$ were used.

comonomers and AIBN was sonicated at $60{ }^{\circ} \mathrm{C}$ for $10 \mathrm{~min}$ and then the glass mold (cylindrical with flat bottom, $10 \mathrm{~mm}$ internal diameter and 2-cm height) was kept in an oven at $90{ }^{\circ} \mathrm{C}$ for $24 \mathrm{~h}$. Time and temperature of reaction were modified to get different conversions. The hydrogels were removed from the glass molds and cut in cylinders of $0.7-1 \mathrm{~cm}$ height. The first compression measurement (as-synthesized test) was then carried out. Afterwards, the hydrogel was washed repeatedly with deionized water, replacing water from time to time and analyzing it spectrophotometrically to detect the end of the extraction of soluble material, in particular, of residual comonomers. Once washed, the specimen was immersed in the desired swelling medium during at least one week and the new compression measurement was performed. In some cases, the hydrogel was then swollen in a different medium to afterwards perform a new compression measurement. Otherwise, it was washed again with deionized water and dried in oven at $80{ }^{\circ} \mathrm{C}$ during $24 \mathrm{~h}$ to determine the weight of the xerogel.

Table 1 summarizes the synthetic conditions employed for each sample. Three parameters of the feed mixture were employed in denoting hydrogel samples: the total monomers concentration $C \mathrm{~T}$ (around 40 or $25 \mathrm{in} \mathrm{g} / 100 \mathrm{~mL}$ or $\% \mathrm{w} / \mathrm{v}$ ), the cross-linker ratio $C$ (around 2 w/w \% BA in the comonomer mixture) and $f_{\mathrm{SS}}$, the SS mole fraction in the comonomer mixture (around 4\% for the unique PVI-SS sample). The symbols used are, as in previous reports, $\mathrm{PVI}_{\mathrm{T}}(C), \mathrm{PSSC}_{\mathrm{T}}(C)$, and PVI-SSC $C_{\mathrm{T}}(C) f_{\mathrm{SS}}$. Several specimens of each sample obtained at variable conversions were studied.

\section{Measurements}

Uniaxial compression measurements were performed on gel cylinders by means of a Microtest (Spain) tensile testing machine at room temperature, using a load cell of $\pm 0.01 N$ sensibility and $50 N$ maximum load. ${ }^{9}$ The compression probe consisted of a pair of parallel Teflon plates to minimize the appearance of shear stresses on the surface of the samples under uniaxial compression. Due to the very small modulus of these soft materials, the initial length was determined as the separation between the upper and lower probes at which the load cell detected a signal above electronic noise. The initial diameter was determined with caliper and the length of the specimens under compression was measured with an optical ruler, with a resolution of $\pm 1 \mu \mathrm{m}$. Once the upper probe was located over the surface of the sample, the load was applied in successive steps of $0.02 \mathrm{~N}$ and the lecture of force and length was done after the relaxation time (about $10 \mathrm{~s}$ ) required to attain stabilized lectures. The absence of hysteresis in 
subsequent loading-unloading tests supports the adequacy of the procedure. Measurements were conducted up to a maximum $20 \%$ compression. Samples were weighed before and after compression measurements and no significant water loss was observed in any case. No barreling effects were observed during compression. One drop of water was deposited on the gel prior to the measurement to lubricate contact with the plate.

Swelling measurements were performed gravimetrically following the methods described elsewhere. $^{3,29,30}$ The polymer volume fraction in the equilibrium swollen gel, $v_{2}$, was determined from the mass of the swollen sample, $m_{\mathrm{h}}$, and that of the dry one, $m_{\mathrm{o}}$, as

$$
v_{2}=\left(1+\rho_{2} S / \rho_{1}\right)^{-1}
$$

where $S$, the degree of swelling, is the ratio of the mass of swelling solvent $\left(m_{\mathrm{h}}-m_{\mathrm{o}}\right)$, to that of dry gel $\left(S=\left(m_{\mathrm{h}}-m_{\mathrm{o}}\right) / m_{\mathrm{o}}\right)$ and $\rho_{2}$ and $\rho_{1}$ represent the density of the dry gel and the swelling medium, respectively. $v_{2 \mathrm{r}}$, the polymer volume fraction in the relaxed network state, that is, when the crosslinkages were introduced (also called memory parameter) was determined from the mass of the assynthesized sample, $m_{\mathrm{hr}}$ instead of $m_{\mathrm{h}}$. Xerogel densities were measured with a Micrometrics 1305 Multivolume Helium Pycnometer.

$\mathrm{pH}$ measurements were carried out with a Corning $245 \mathrm{pHmeter}$, at room temperature. Calibration was made with standard buffer solutions from Carlo Erba. The degree of protonation, $\alpha$, was determined by measuring the initial $\mathrm{pH}_{\mathrm{i}}$ of the bath before immersion of the gel and the final one, $\mathrm{pH}_{\mathrm{f}}$, reached in the bath at equilibrium, ${ }^{28}$

$$
\alpha=\frac{10^{-\mathrm{pH}_{\mathrm{i}}}-10^{-\mathrm{pH}_{\mathrm{f}}}}{C_{\text {ef }}}
$$

where $C_{\text {ef }}$ represents the effective gel concentration, that is, the moles of monomer units in the gel, per unit volume of the bath.

\section{RESULTS AND DISCUSSION}

Compression measurements were analyzed through plots of the applied stress, $\tau,\left(\tau=f / A_{\mathrm{S}}\right.$ with $f$ being the acting force and $A_{\mathrm{S}}$ the cross section of the undeformed swollen specimen) versus $\lambda-\lambda^{-2}$, where $\lambda=L / L_{\mathrm{o}}$ is the relative deformation caused by compression of the length of the swollen sample along the direction of the stress $(L)$, with respect to the initial length $\left(L_{\mathrm{o}}\right)$ of the swollen but undistorted sample. They show the typical curvature at the smallest range of deformation that is generally assigned to imperfect geometry of the surface of the gel specimen. ${ }^{9,14,22,35-37}$ The usual correction for such curvature was applied..$^{9}$ All corrected measurements show linear behavior and the elastic modulus, $G$, was determined, by linear regression, as the slope of such plots. The corrected elastic modulus, $G$, increases in about $10 \%$ with respect to the uncorrected value. In the following, only corrected values will be presented.

Several measurements were performed on assynthesized specimens to check their reproducibility. No significant hysteresis was observed for all the as-synthesized specimens here studied, that is to say, the elastic modulus was about the same in consecutive loading-unloading experiments. It was also observed that consecutive loading measurements on a given specimen, and measurements on different specimens synthesized with the same feed conditions, yield the same corrected elastic modulus within 6\% experimental uncertainty.

For homogeneous networks of Gaussian chains, the elastic modulus $G$ of a swollen specimen is related to the network chain density (also called cross-linking density), $v_{\mathrm{e}}$, by the equation ${ }^{38}$

$$
G=A_{\phi} R T v_{\mathrm{e}} v_{2 r}^{2 / 3} v_{2}^{1 / 3}
$$

with $A_{\phi}=1$ for affine networks, $A_{\phi}=1-2 / \phi$ for phantom networks (better suited for swollen samples) and $\phi$, (the cross-linker functionality) $=4$ for BA. The molecular weight of chains between consecutive knots is $M_{\mathrm{c}}=\rho_{2} / v_{\mathrm{e}}$. Particularizing eq 3 for the as-synthesized samples it results. ${ }^{22}$

$$
G_{o}=A_{\phi} R T v_{\mathrm{e}} v_{2 r}
$$

Average values of two $G_{0}$ determinations differing always less than $6 \%$ are given in Table 2. The cross-linking densities and $M_{\mathrm{c}}$ were calculated from $G_{\mathrm{o}}$ values (eq 4 with affine model) and appear summarized in Table 2. The cross-linking density of PVI40(2) is around $65 \mathrm{~mol} / \mathrm{m}^{3}$ while that of PVI25(2) is around $30 \mathrm{~mol} / \mathrm{m}^{3}$, that is, $v_{\mathrm{e}}$ increases with $C_{\mathrm{T}}$, as previously observed for other types of $v_{\mathrm{e}}$ measurements. ${ }^{5,18,25,39}$

For samples synthesized up to total conversion, the model of polymer network with pendant vinyl groups that Bromberg et al. ${ }^{39}$ proposed, predicts that the cross-linking density is proportional to $C_{\mathrm{T}} \times[\mathrm{BA}]:$

$$
v_{e}(P V)=2 a^{6} C_{\mathrm{T}}[\mathrm{BA}] \rho_{2} / M_{\mathrm{o}}
$$


Table 2. Corrected Elastic Modulus $\left(G_{o}\right)$ Measured on As-Synthesized Samples, Molecular Weight of Chains between Consecutive Knots $\left(M_{c}\right)$ Determined with the Given Xerogel Densities $\left(\rho_{2}\right)$ and Cross-Linking Density $\left(v_{\mathrm{e}}\right)$ Determined from Compression Measurements with the Affine Model

\begin{tabular}{|c|c|c|c|c|c|c|}
\hline Sample & Specimen & $G_{\mathrm{o}}(\mathrm{kPa})$ & $\rho_{2}\left(\mathrm{~g} / \mathrm{cm}^{3}\right)$ & $M_{\mathrm{c}}(\mathrm{g} / \mathrm{mol})$ & $v_{\mathrm{e}}\left(\mathrm{mol} / \mathrm{m}^{3}\right)$ & $v_{\mathrm{e}}(P V)\left(\mathrm{mol} / \mathrm{m}^{3}\right)$ \\
\hline \multirow[t]{5}{*}{ PVI25(2) } & S9 & 11.0 & \multirow[t]{5}{*}{1.239} & $35 \times 10^{3}$ & 35 & 22 \\
\hline & S28 & 12.1 & & $46 \times 10^{3}$ & 27 & 23 \\
\hline & S29 & 12.6 & & $46 \times 10^{3}$ & 27 & 22 \\
\hline & S30 & 11.8 & & $46 \times 10^{3}$ & 27 & 23 \\
\hline & S31 & 12.4 & & $41 \times 10^{3}$ & 30 & 22 \\
\hline \multirow[t]{4}{*}{ PVI40(2) } & $\mathrm{S} 3$ & 47.5 & \multirow[t]{4}{*}{1.25} & $18 \times 10^{3}$ & 69 & 53 \\
\hline & $\mathrm{S} 25$ & 43.2 & & $19 \times 10^{3}$ & 65 & 55 \\
\hline & $\mathrm{S} 26$ & - & & - & - & 58 \\
\hline & S27 & - & & - & - & 56 \\
\hline \multirow[t]{2}{*}{ PSS40(2) } & S8 & 2.3 & \multirow[t]{2}{*}{1.29} & $350 \times 10^{3}$ & 3.7 & 28 \\
\hline & S13 & 3.6 & & $215 \times 10^{3}$ & 6.0 & 25 \\
\hline PVI-SS40(2)4 & $\mathrm{S} 23$ & 34.5 & 1.315 & $14 \times 10^{3}$ & 93 & 62 \\
\hline
\end{tabular}

The cross-linking density calculated with the model of polymer network with pendant vinyl groups, $v_{\mathrm{e}}(P V)$, is also listed.

where $a^{6}$ equals the product of the molar volumes of bifunctional monomer and crosslinker $(0.00964$ $\mathrm{L}^{2} / \mathrm{mol}^{2}$ for PVI40(2), $0.00973 \mathrm{~L}^{2} / \mathrm{mol}^{2}$ for PVI25(2), $0.0205 \mathrm{~L}^{2} / \mathrm{mol}^{2}$ for PSS40(2), and $0.01007 \mathrm{~L}^{2} / \mathrm{mol}^{2}$ for PVI-SS40(2)4). Equation 5 predicts correctly the permanent cross-linking density of hydrogels..$^{5,18,25,39}$ The $v_{\mathrm{e}}(P V)$ calculated values, can be compared with the $v_{\mathrm{e}}$ determined through compression measurements, in Table 2.

Several $v_{\mathrm{e}}$ results are remarkable. $v_{\mathrm{e}}(P V)$ values are always larger and not too different from $v_{\mathrm{e}}$, in coherence with previous results on the descent of $v_{\mathrm{e}}$ with increasing conversion owing to the crosslinker incorporation mainly in the first stages of postgel reactions. ${ }^{18}$ However, there is a significant exception, PSS. This polyelectrolyte does not achieve gelation with AIBN, benzoylperoxide or potassium persulfate as initiators, only lightly cross-linked specimens were obtained. This result reveals that $C_{\mathrm{T}}$ and [BA] are not too far from the threshold for gelation of PSS. The low cross-linking efficiency of PSS is likely due to the low reactivity of SS with the crosslinker, BA. ${ }^{12,40}$

Figures 1-4 compare the stress-corrected strain results of several samples as-synthesized and swollen to different degrees with (Figs. 2, 3, and 4) or without ionization (Fig. 1), and Table 3 compiles the corresponding $G$ values. Average values of two consecutive measurements differing always less than 6\% are given. Swelling in deionized water gives place to neutral PVI gels and, as shown in Figure 1, the elastic modulus scarcely changes with respect to the corresponding assynthesized sample. The elastic modulus of polyelectrolyte gels has received great attention. ${ }^{11,13,15,41-47}$ Here, three types of ionic gels were studied, (i) the highly charged PSS polyelectrolyte gel (Fig. 2), (ii) PVI partially protonated by swelling in acid aqueous solutions (Fig. 3) and (iii) PVI-SS polyampholyte (Fig. 4) with negative fixed charges (sulfonic groups) and positive charges (protonated of imidazole groups). ${ }^{3}$ Only as-synthesized specimens of PSS were measured because throughout swelling these samples disintegrate.

A pronounced hysteresis of PVI40(2)-S26 swollen in $\mathrm{HCl}$ aqueous solution (initial $\mathrm{pH}=2.60$, final $\mathrm{pH}=3.66$ and $17 \%$ degree of ionization) can be observed in Figure 3. However, the similar sample PVI40(2)-S27 swollen in an aqueous solution containing $\mathrm{HCl}$ (initial $\mathrm{pH}=2.45$, final $\mathrm{pH}=$ 5.60 and $13 \%$ degree of ionization) and $1.0 \mathrm{M}$ $\mathrm{NaCl}$, gives reproducible consecutive measurements and the same holds for PVI40(2)-S3 swollen in $\mathrm{H}_{2} \mathrm{SO}_{4}$ aqueous solution (initial $\mathrm{pH}=2.50$, final $\mathrm{pH}=4.06$ and $29 \%$ degree of ionization). Hysteresis of ionic gels evidences that loading induces changes of the polymer network structure, which might be attributed to strain-induced ionic redistribution. ${ }^{15}$ Protonated rings are randomly distributed along the polymer network to minimize the energy due to electrostatic interactions but, upon loading, their distribution may change by proton migration towards a new configuration of minimum energy. Such redistribution of charges anchored to the polymer skeleton is not 

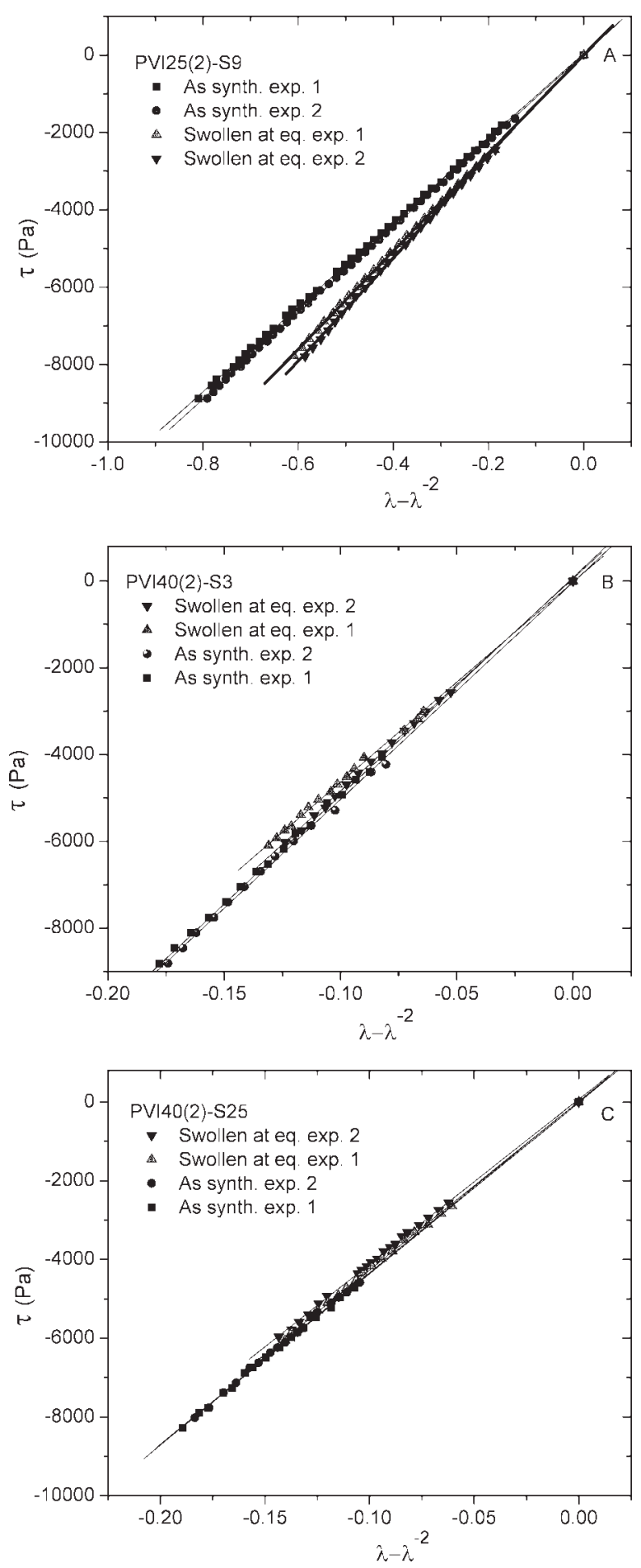

Figure 1. Compression stress measurements (loading followed by unloading) performed on several PVI samples as-synthesized and swollen at equilibrium in deionized water. (A) PVI25(2)-S9, (B) PVI40(2)-S3, and (C) PVI40(2)-S25.

possible in ionic gels with strong acid or basic dissociated functionalities, whose positions are fixed during copolymerization. Ionic strength provided

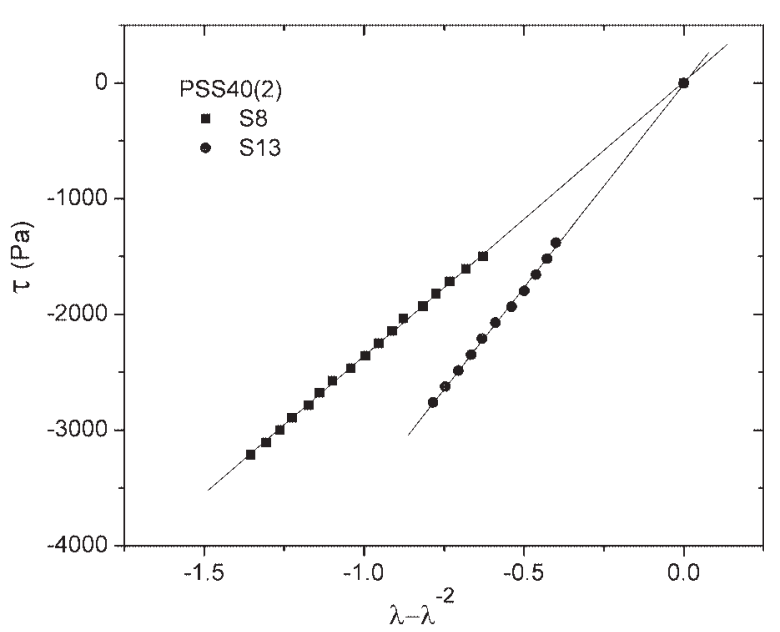

Figure 2. Compression stress measurements performed on two PSS40(2) specimens as-synthesized.

by salt or by divalent sulfate ions, shields the electrostatic interactions making less significant the ionic redistribution. The second measurement (much larger slope) was discarded in determining the $G$ value of PVI40(2)-S26 given in Table 3.

The influence of salt on $G$ may be observed in comparing the behavior of S26 and S27 of PVI40(2). The elastic modulus of PVI40(2) protonated to $15 \%$, is about $10 \%$ larger with salt. It does not represent a large influence and therefore it may be concluded that the presence of $\mathrm{NaCl}$ does not change the mechanical properties of the polymer networks significantly, ${ }^{45}$ in spite of the dramatic influence on swelling observed for protonated PVI. ${ }^{4}$

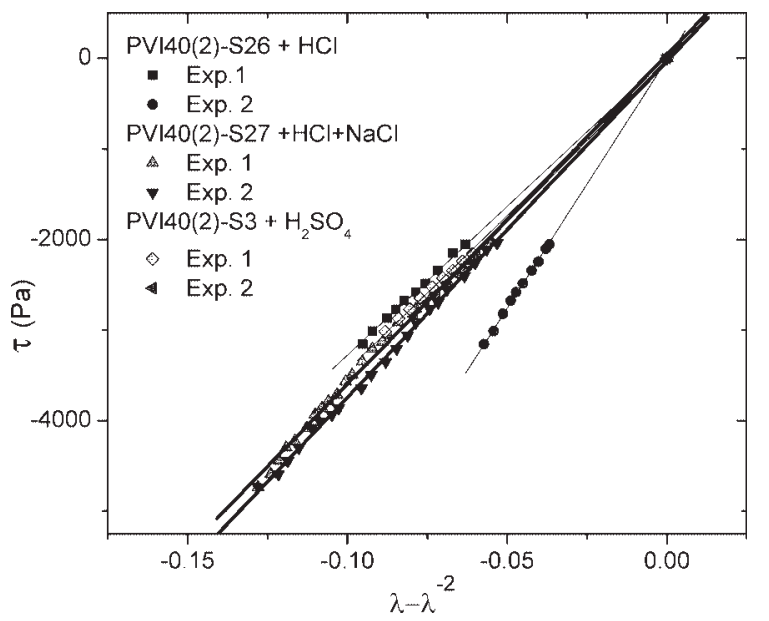

Figure 3. Compression stress measurements (loading followed by unloading) performed on several PVI40(2) specimens swollen in acid aqueous solutions to different degrees of protonation. 


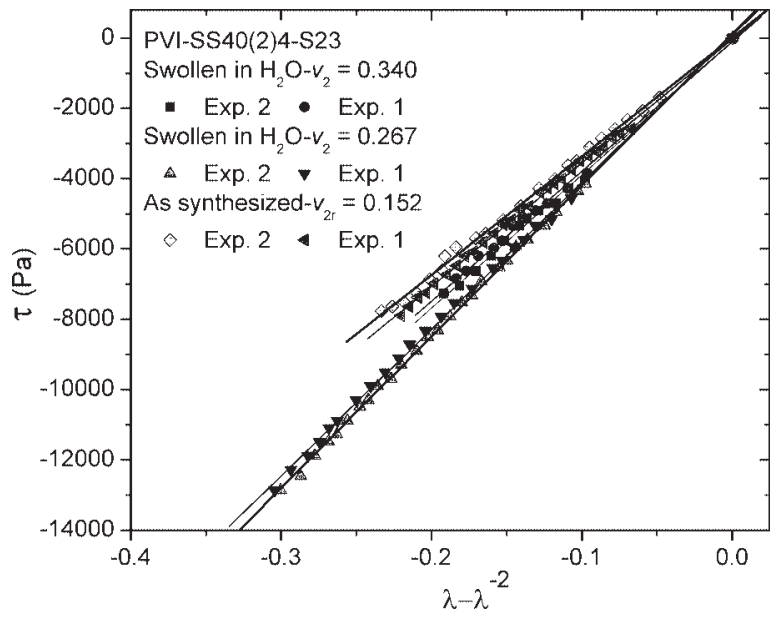

Figure 4. Compression stress measurements (loading followed by unloading) performed on PVISS40(2)4 swollen in deionized water. Ionization is due to the presence of $4 \%$ (in moles) sodium styrenesulfonate moieties and to partial protonation of imidazole.

Three measurements were performed on the polyampholyte PVI-SS, as-synthesized and swollen up to two different degrees. It was previously found that PVI-SS samples of amphoteric character experience a pronounced overshooting effect upon swelling in deionized water, that is, immediately after immersion of the as-synthesized specimen, swelling goes rapidly to a maximum value and from then on it decreases to reach equilibrium. ${ }^{29,30}$ Correspondingly, $v_{2}$ passes through a minimum, as shown for PVI-SS40(2)4 in Figure 5, where the arrows mark the precise $v_{2}$ values at which compression measurements were performed. The formation of ionic crosslinks or intermolecular salt-linkages seems to dominate the hardness of some amphoteric gels ${ }^{47}$ but that is not the case for PVI-SS, as shown below. Over-

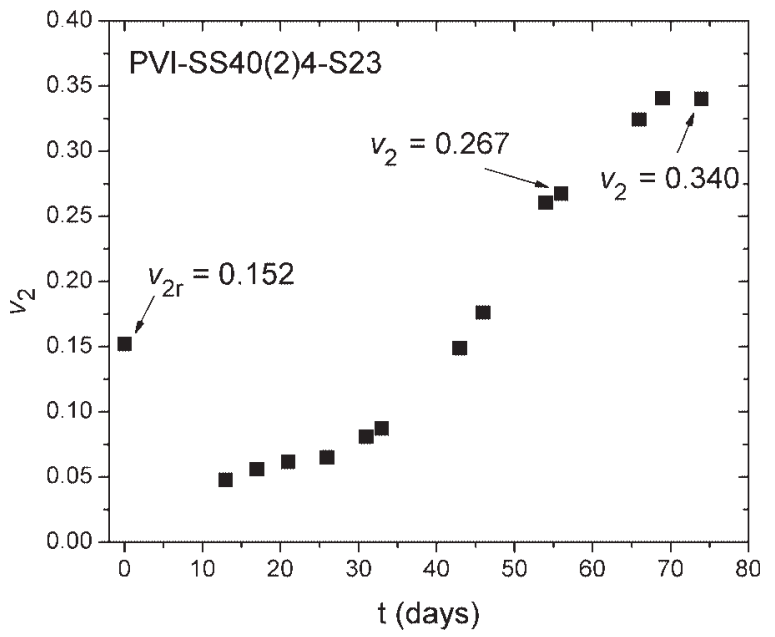

Figure 5. Time evolution of the polymer volume fraction. Arrows mark the time at which compression measurements shown in Figure 4 were performed.

shootings may be due to several phenomena, for example, to post-crosslinking reactions taking place during swelling; in such case, overshooting is usually accompanied by an enlargement of $G{ }^{46}$ However, if the overshooting is associated with the loss of mechanical integrity caused by removal of weakly crosslinked material during a prolonged immersion, $G$ is reduced..$^{10}$ The overshooting effect of PVI-SS was assigned to ion exchange ${ }^{30}$ and it seems to decrease slightly $G$ values.

An unexpected result regarding Tables 2 and 3, is that the elastic moduli are not directly related to $v_{2}$; for example, $G_{\mathrm{o}}$ values are not always larger than $G$ values of the same sample washed and swollen in water or acid aqueous solutions. This behavior was also observed in some previous reports. ${ }^{22,48}$ The Gaussian theory has no explanation for this result since in accordance with eq 3 ,

Table 3. Elastic Modulus of Several Specimens of Samples PVI40(2), PVI25(2), and PVI-SS40(2)4 Swollen in Different Media, with Different Degree of Protonation (for PVI) or Ionization ( $f_{\text {SS }}$ for PVI-SS) $(\alpha)$ and Polymer Volume Fraction in the Swollen State $\left(v_{2}\right)$

\begin{tabular}{lclcc}
\hline \multicolumn{1}{c}{ Sample } & Swelling Medium & \multicolumn{1}{c}{$v_{2}$} & $\alpha$ & $G(\mathrm{kPa})$ \\
\hline PVI25(2)-S9 & $\mathrm{H}_{2} \mathrm{O}$ & 0.0873 & - & 12.9 \\
PVI40(2)-S3 & $\mathrm{H}_{2} \mathrm{O}$ & 0.159 & - & 50.1 \\
& $\mathrm{H}_{2} \mathrm{SO}_{4} / \mathrm{H}_{2} \mathrm{O}$ & 0.0780 & 0.29 & 35.1 \\
PVI40(2)-S25 & $\mathrm{H}_{2} \mathrm{O}$ & 0.138 & - & 42.3 \\
PVI40(2)-S27 & $\mathrm{HCl} /{\mathrm{NaCl} / \mathrm{H}_{2} \mathrm{O}}_{0.106}$ & 0.13 & 36.8 \\
PVI40(2)-S26 & $\mathrm{HCl} / \mathrm{H}_{2} \mathrm{O}$ & 0.0591 & 0.17 & 32.9 \\
PVI-SS40(2)4-S23 & $\mathrm{H}_{2} \mathrm{O}$ int. & 0.267 & 0.04 & 42.4 \\
& $\mathrm{H}_{2} \mathrm{O}$ eq. & 0.340 & 0.04 & 37.3
\end{tabular}


as swelling increases (decreasing $v_{2}$ ), $G$ should decrease.

For highly swollen hydrogels the shortest network chains become highly extended and deviation from the Gaussian statistics may appear owing to the finite extensibility of the network chains. ${ }^{11,41,42}$ The extent of Gaussian behavior depends on the chemical nature of the polymer (that is, on chain stiffness), on the average length of chains between crosslinks and on its polydispersity. PVI is rather rigid and that favors the appareance of non-Gaussian effects even with low degrees of swelling. Moreover, several evidences previously published, support the non-Gaussian character of PVI swollen hydrogels. MooneyRivlin plots of the reduced force are linear for assynthesized samples while they are U shaped for protonated PVI and upwards curvature was ascribed to non-Gaussian effects. ${ }^{9}$ Additionally, quantitative analysis of the swelling data of PVISS and protonated PVI requires the consideration of a non-Gaussian parameter that increases with the degree of ionization. . $^{3,4}$

Let us call $\beta$ the phenomenological parameter that takes into account the non-Gaussian effects on $G$, in such a way that

$$
G=A_{\phi} R T \beta v_{\mathrm{e}} v_{2 r}^{2 / 3} v_{2}^{1 / 3}
$$

with $\beta=1$ for Gaussian systems and $\beta>1$ (increasing with swelling) for systems showing departures from the Gaussian model. As-synthesized samples with chains between knots containing more than 100 average monomer units (as it is the case for all the samples here studied) are assumed to be Gaussian.

Swelling has three opposite effects on the elastic modulus, which are related with the following three factors in eq 6: $v_{2}, \beta$ and $A_{\phi}$. On the one hand, $v_{2}$ decreases regarding $v_{2 \mathrm{r}}$ and therefore the modulus decreases with respect to that of the assynthesized sample. On the other, chain extension increases $\beta$ and that increases $G$. Furthermore, upon swelling the sample shifts from the affine limit to the phantom one and $A_{\phi}$ decreases from 1 to $1-2 / \phi=1 / 2$ for a tetrafunctional cross-linker as BA. Real samples are in-between the affine and phantom limits. The affine model applies better to samples in which the fluctuations of knots are suppressed by transient interactions between network chains. It is favored in less swollen and less deformed samples, that is, for as-synthesized samples. Upon swelling, the chains between cross- links become stretched (the same as under extensional stress), the constraints for knots fluctuation diminish and the sample approaches the phantom limit

With these premises and since cross-linking density is a structural characteristic that does not change upon swelling, combining eqs 4 and 6 it results

$$
\beta=2 \frac{G}{G_{0}}\left(\frac{v_{2 r}}{v_{2}}\right)^{1 / 3}
$$

Figure 6 presents the non-Gaussian parameter $\beta$ for several samples swollen up to different extents, with or without protonation. $G_{\mathrm{o}}$ was not measured for S26 and S27 of PVI40(2) and $\beta$ was calculated with $v_{\mathrm{e}}(P V)$ values. Figure 6 shows that $\beta$ increases with swelling and levels off for the largest $1 / v_{2}$ values. All the $\beta$ values fall into a single curve except for PVI swollen in a diprotic acid solution. On the light of a qualitative interpretation of the swelling results, it was initially postulated that divalent anions might induce the formation of ionic cross-links ${ }^{26}$ between a sulfate anion and two protonated imidazole moieties. However it must be discarded because that would decrease the length of chains between knots (increasing $\beta$ ) and it could also increase $v_{\mathrm{e}}$, which would rebound in, apparently, increasing $\beta$ too. Divalent counterions are related to the formation of linearly aligned regions which affect the rigidity, ${ }^{49}$ but it would also contribute to rise $\beta$ with respect to univalent counterions. The reason for the low $\beta$ value of PVI swollen in a diprotic acid solution is not clear at the moment.

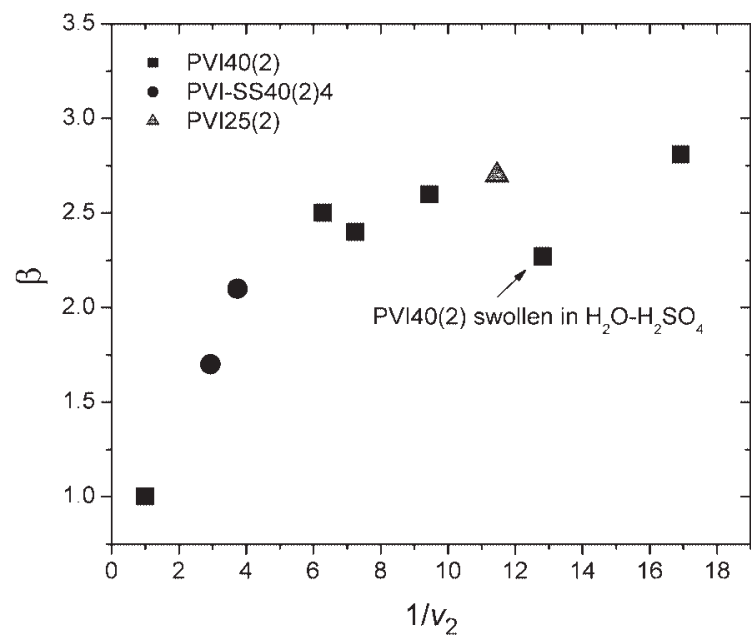

Figure 6. Non-Gaussian parameter derived from compression measurements. 
Ionic crosslinks must also be discarded for PVI-SS since their $\beta$ values are as large as expected for the degree of swelling achieved at any overshooting stage. If the attractive interactions between sulfonate and protonated imidazole ions, were strong enough to be considered as ionic cross-links, $\beta$ should be larger. This result is in good accordance with previous quantitative analysis of the antipolyelectrolyte effect of this same amphoteric sample, which concluded that the concentration of mobile counterions was not proportional to the net fixed charge, but to the addition of cationic and anionic side groups, what discards the formation of interchain ionic pairs. ${ }^{3}$

\section{CONCLUSIONS}

The compression modulus of several hydrogels based on $N$-vinylimidazole was determined. These hydrogels were neutral, amphoteric or cationic and their ionic character came from protonation of imidazole groups and from dissociation of sulfonic groups. Compression-strain measurements were performed on samples as-synthesized and swollen in deionized water or in acid aqueous solutions, with and without salt. It was thus found that $v_{\mathrm{e}}$ determined on as-synthesized samples obtained at high conversions, are in good accordance with $v_{\mathrm{e}}(P V)$ calculated by means of the model of polymer networks with pendant vinyl groups. A peculiar system is poly(sodium styrenesulfonate) whose cross-linking density is much lower than expected, likely due to the low reactivity of SS with the cross-linker.

A non-Gaussian parameter $(\beta)$ was introduced to explain that the elastic moduli of samples swollen at equilibrium are larger than predicted by the Gaussian model. The $\beta$ values of the several systems studied, increase with swelling and fall in a single curve. The only exception is protonated PVI with divalent counteranions for which, the formation of ionic crosslinks between a counteranion and two protonated moieties placed in different chains was discarded, because such ionic crosslinks would contribute to enlarge $\beta$. The possible contribution to $v_{\mathrm{e}}$ of ionic crosslinks formed by electrostatic interaction between sulfonate and protonated imidazole groups, was also discarded for amphoteric PVI-SS. The common behavior regarding rigidity $(\beta)$ of neutral and slightly ionic gels based on $N$-vinylimidazole, indicates that the neutral part dominates the elastic properties of weakly charged gels.
In summary, swelling has two opposite effects on $G$; on the one hand $G$ decreases because the polymer volume fraction diminish and the system shifts from the affine limit to the phantom one; on the other, $\beta$ increases and contributes to increasing $G$. The balance of those two opposite effects determines the variation of $G$ with swelling.

Financial support from DGI (Spain) under grant CTQ2007-61007/BQU is gratefully acknowledged.

\section{REFERENCES AND NOTES}

1. Turan, E.; Demirci, S.; Caykara, T. J Polym Sci Part B: Polym Phys 2008, 46, 1713-1724.

2. Tokuyama, H.; Ishihara, N.; Sakohara, S. Eur Polym J 2007, 43, 4975-4982.

3. Valencia, J.; Pierola, I. F. J Polym Sci Part B: Polym Phys 2007, 45, 1683-1693.

4. Molina, M. J.; Gomez-Anton, M. R.; Pierola, I. F. J Phys Chem B 2007, 111, 12066-12074.

5. Pacios, I. E.; Pierola, I. F., J Appl Polym Sci 2009, 112, 1579-1586.

6. Baselga, J.; Fuentes, I. H.; Pierola, I. F.; Llorente, M. A. Macromolecules 1987, 20, 3060-3065.

7. Atta, A. M.; Arndt, K. F. J Appl Polym Sci 2005, 97, 80-91.

8. Guilherme, M. R.; Campese, G. M.; Radovanovic, E.; Rubira, A. F.; Feitosa, J. P. A.; Muñiz, E. C. Polymer 2005, 46, 7867-7873.

9. Valencia, J.; Baselga, J.; Pacios, I. E.; Pierola, I. F. Macromol Symp 2003, 200, 235-242.

10. Lee, J. H.; Bucknall, D. G. J Polym Sci Part B: Polym Phys 2008, 46, 1450-1462.

11. Nisato, G.; Munch, J. P.; Candau, S. J. Langmuir 1999, 15, 4236-4244.

12. Xue, W.; Huglin, M. B.; Jones, T. G. Eur Polym J 2005, 41, 239-248.

13. Horkay, F.; Han, M.-H.; Han, I. S.; Bang, I.-S.; Magda, J. J Polymer 2006, 47, 7335-7338.

14. Muniz, E. C.; Geuskens, G. Macromolecules 2001, 34, 4480-4484.

15. Miquelard-Garnier, G.; Creton, C.; Hourdet, D. Soft Matter 2008, 4, 1011-1023.

16. Kioussis, D. R.; Kofinas, P. Polymer 2005, 46, 10167-10172.

17. Sedlakova, Z.; Bouchal, K.; Ilavsky, M. Polym Bull 2000, 44, 585-592.

18. Pacios, I. E.; Pierola, I. F. Macromolecules 2006, 39, 4120-4127.

19. DiMarzio, E. A. J Res Natl Bur Stand 1964, 68A, 611.

20. Valentin, J. L.; Carretero-Gonzalez, J; MoraBarrantes, I.; Chasse, W.; Saalwachter, K. Macromolecules 2008, 41, 4717-4729. 
21. Gauthier, M. A.; Luo, J.; Calvet, D.; Ni, C.; Zhu, X. X.; Garon, M.; Buschmann, M. D. Polymer 2004, 45, 8201-8210.

22. Okay, O.; Durmaz, S. Polymer 2002, 43, 12151221.

23. Davis, T. P.; Huglin, M. B. Makromol Chem 1991, 189, 195-205.

24. Ikehata, A.; Ushiki, H. Polymer 2002, 43, 20892094.

25. Pacios, I. E.; Molina, M. J.; Gomez-Anton, M. R.; Pierola, I. F. J Appl Polym Sci 2007, 103, 263269.

26. Molina, M. J.; Gomez-Anton, M. R.; Pierola, I. F. Macromol Chem Phys 2002, 203, 2075-2082.

27. Molina, M. J.; Gomez-Anton, M. R.; Pierola, I. F. J Polym Sci Part B: Polym Phys 2004, 42, 22942307.

28. Horta, A.; Molina, M. J.; Gomez-Anton, M. R.; Pierola, I. F. J Phys Chem B 2008, 112, 10123; Correction J Phys Chem B 2008, 112, 13166.

29. Valencia, J.; Pierola, I. F. Eur Polym J 2001, 37, 2345-2352.

30. Valencia, J.; Pierola, I. F. J Appl Polym Sci 2002, 83, 191-200.

31. Das, M.; Kumacheva, E. Colloid Polym Sci 2006, 284, 1073-1084.

32. Pich, A.; Tessier, A.; Boyko, V.; Lu, Y.; Adler, H.-J P Macromolecules 2006, 39, 7701-7707.

33. Lee, W.-F.; Hsu, Ch-H. J Appl Polym Sci 1999, 74, 3242-3253.
34. Isik, B. Adv Polym Tech 2003, 22, 246-251.

35. Rietsch, F.; Froelich, D. Polymer 1975, 16, 873878.

36. Gutowska, A.; Bae, Y. H.; Jacobs, H.; Feijen, J.; Kim, S W Macromolecules 1994, 27, 4167-4175.

37. Sayil, C.; Okay, O. Polymer 2001, 42, 7639-7652.

38. Flory P. J Principles of Polymer Chemistry; Cornell University Press: Ithaca, NY, 1953.

39. Bromberg, L.; Grosberg, A. Y.; Matsuo, E. S.; Suzuki, Y.; Tanaka, T. J Chem Phys 1997, 106, 2906-2910.

40. Baselga, J.; Llorente, M. A.; Nieto, J. L.; Hernandez-Fuentes, I.; Pierola I. F. Eur Polym J 1988, 24, 161-165.

41. Gundogan, N.; Melekaslan, D.; Okay, O. J Appl Polym Sci 2004, 94, 135-141.

42. Treloar, L. R. G. The Physics of Rubber Elasticity; Oxford University Press: Oxford, UK, 1975.

43. Rubinstein, M.; Colby, R. H.; Dobrynin, A. V.; Joanny, J F. Macromolecules 1996, 29, 398-406.

44. Hasa, J.; Ilavsky, M.; Dusek, K. J Polym Sci Polym Phys Ed 1975, 13, 253-262.

45. Erdal, S.; Bahar, I.; Erman, B. Polymer 1998, 39, 2035-2041.

46. Durmaz, S.; Fank, S.; Okay, O. Macromol Chem Phys 2002, 203, 663-672.

47. Tamagawa, H.; Nogata, F.; Yagasaki, K. J Colloid Interface Sci 2004, 275, 107-112.

48. Luo, L. B.; Kato, M.; Tsuruta, T.; Kataoka, K.; Nagasaki, Y. Macromolecules 2000, 33, 4992-4994.

49. Horkay, F.; Basser, P. J. J Polym Sci Part B: Polym Phys 2008, 46, 2803-2810. 